\title{
Obstructive Sleep Apnea Syndrome: An Under-recognized Clinical Entity with Significant Systemic Morbidities
}

\author{
Ramakant Dixit ${ }^{1}$, Satyadeep Verma ${ }^{2}$, Jitendra Jalutharia ${ }^{3}$ and Anubhav Sharma ${ }^{4}$ \\ ${ }^{1}$ Professor, ${ }^{3}$ Resident, ${ }^{4}$ Senior Resident, Department of Respiratory Medicine and Tuberculosis, \\ JLN Medical College and Associated Group of Hospitals, Ajmer, Rajasthan; ${ }^{2}$ Senior Resident, \\ Department of Respiratory Medicine and Tuberculosis, RUHS College of Medical Sciences \\ and Associated Hospital, Jaipur, Rajasthan, India
}

\begin{abstract}
Obstructive sleep apnea syndrome (OSAS) is a highly prevalent yet under recognized sleep disorder, characterized by repeated disruptions of breathing during sleep. Not just merely a local phenomenon of upper respiratory tract obstruction, this has many consequences that includes intermittent hypoxia, intermittent hypercapnia, reoxygenation, intra thoracic pressure changes, sympathetic activation, micro-awakenings and sleep fragmentation leading to metabolic dysregulation, endothelial dysfunction, systemic inflammation, oxidative stress, hypercoagulation, and neurohumoral changes that causes excessive daytime sleepiness, neurocognitive deterioration, endocrine, metabolic and other systemic effects including poor quality of life. There are increasing evidences to suggest that OSAS is a systemic inflammatory disease. Epidemiological studies have identified OSAS as an independent risk factor in cardiovascular diseases, metabolic syndrome and bronchial asthma. Patho-physiological changes in OSAS are causally linked to the hypertension with increase risk for heart failure, stroke and cardiovascular mortality. In addition, OSAS is associated with several other disorders and comorbidities that may affect almost every organ systems of our body. Despite having many complex systemic consequences, OSAS and its secondary effects are mostly controllable by continuous positive air pressure (CPAP). This disorder largely remains an underestimated clinical entity due to unawareness by both the patient and physician alike. There is need for awareness regarding this entity among all specialties of
\end{abstract}

medicine and surgery as the so called undetected/occult disease if remain untreated may lead to significant systemic morbidities and at times mortality.

\section{INTRODUCTION}

Sleep is essential to life and to overall health. Most of us need an adequate and quality sleep to function in a proper manner. Lack of sleep can have profound consequences on a daily routine and potentially long term basis for our health and mental well being. Long term health consequences related to lack of sleep could be high blood pressure, diabetes, obesity and heart diseases that may culminate to a shortened life expectancy. Most common types of sleep disorders are sleep apnea, insomnia, narcolepsy, restless leg syndrome, sleep paralysis etc. Among all the sleep disorders, sleep apnea is most common type of disorder. It is of three types- obstructive sleep apnea, central sleep apnea and mixed sleep apnea. Obstructive sleep apnea is most common among these three and rather a complex syndrome called obstructive sleep apnea syndrome (OSAS) in view of its wide clinical manifestations and systemic consequences.

OSAS is a highly prevalent yet under-recognized clinical problem. It is a potentially disabling condition characterized by disruptive snoring, repeated episodes of complete or partial pharyngeal obstruction during sleep, marked swings in intra thoracic pressure and increased sympathetic activities resulting in nocturnal hypoxemia, frequent arousals, witnessed nocturnal interruptions and excessive daytime sleepiness. ${ }^{1}$ OSAS has become a leading public health problem both in the developed and developing nations. However, awareness regarding its 
existence, diagnostic options, management and consequences of untreated OSAS largely remains inadequate. In developing countries, the burden is enormous as resources for adequate sleep medicine facilities for diagnosis are grossly lacking. Therefore, there is always a need for low cost, simple and accurate diagnostic and therapeutic modalities for true estimation of disease burden and subsequent management.

In the Western world, the prevalence of OSAS is 3\% to $7 \%$ in men and $2 \%$ to $5 \%$ in women. ${ }^{2,3}$ In Indian scenario $2.4 \%$ to $4.9 \%$ male and $1 \%$ to $2 \%$ female are affected by OSAS as per data available from few centres. ${ }^{4}$ Polysomnography (PSG) is considered as gold standard for diagnosing sleep disorders that essentially include either home based or in hospital overnight recording and assessment of various sleep parameters using Polysomnography. The severity of OSAS is classified as mild, moderate and severe based upon apnea-hypopnea index (AHI). The management options of OSAS include behavior modification, sleep position changes, weight control, continuous positive air pressure (CPAP), oral appliances and surgery etc. The CPAP remains the time trusted and most effective measure among all.

There are enough evidences today to suggest that OSAS is not a localized disorder rather it has several significant systemic consequences that are usually underestimated among the treating physician, pulmonologist, cardiologist, surgeon, pediatricians, neurologist, psychiatrist, endocrinologist and rather all other clinical branches of medicine and surgery because patients usually avoid to express the symptoms as they assume them to be nothing unusual and treating physician also do not ask specifically about them in routine practice. The aim of writing this review is to create awareness among all medical practitioners regarding this significant but unfortunately under suspected clinical entity which at present truly represent the tip of an iceberg.

\section{PATHOPHYSIOLOGY OF OSAS}

A variety of factors have been identified that are known to contribute this condition and these include alteration in the upper airway anatomy, neuromuscular function and sleep-wake and ventilator control instability. The upper airway in OSAS subjects, when compared with controls, is smaller and narrowed laterally rather than in the anteroposterior dimension due to increased thickness of the muscular pharyngeal wall. Nasal obstruction due to either mechanical (septal deviation, enlarged inferior turbinates and nasal polyps) or inflammatory/ vasomotor (acute and chronic rhinitis) causes has been proposed to contribute to OSAS. The frequency of OSAS events may increase in the supine compared with the lateral recumbent posture. Oxygen desaturation may also worsen further in the supine position. This positional variability of OSAS has been attributed possibly to the effects of gravity on upper airway size and shape. ${ }^{5}$ Racial studies and chromosomal mapping, familial studies and twin studies have provided evidence for the possible link between the OSAS and genetic factors also. ${ }^{6}$

\section{RISK FACTORS OF OSAS}

There are certain known and hidden risk factors that can lead to development of OSAS, among them obesity is most common and most prevalent. But it is also true that OSAS is not uncommon in non obese individuals. Other factor includes- old age, male gender, anatomical defect like micrognathia, retrognathia, high arched palate, macroglossia, nasal polyp, deviated nasal septum, genetic predisposition, familial aggregation, cigarette smoking, menopause, alcohol intake, night time nasal congestion, endocrine abnormalities (hypothyroidism/acromegaly, polycystic ovarian syndrome), Down's syndrome, drugs like benzodiazepines, muscle relaxants, testosterone therapy ${ }^{7,8}$ etc.

Recently bronchial asthma, chronic obstructive pulmonary disease (COPD), metabolic syndrome, certain psychiatric problems etc has also emerged as important risk factors for development of OSAS. Poor control of these disorders has been consistently associated with poor sleep, restlessness, breathlessness and poor quality of life among these patients.

\section{OSAS and Respiratory disorders:}

It is primarily a sleep related respiratory disorder where the primary event is sleep-related collapse of the pharynx in the face of persistent ineffective breathing efforts. Repetitive collapse of the upper airway during sleep leads to ineffective sleep and day time sleepiness. The symptoms are therefore related to (i) sleep i.e. unrefreshing sleep, unrestorative sleep, disturbing snoring, breathing pauses, restless sleep, nocturia, nocturnal sweating, gasping sounds, wake-up suffocating (ii) wakefulness i.e. tiredness, lack of energy, sleepiness, 
memory impairment, anxiety and irritability, depression, lack (or loss) of interest, sexual dysfunction (erectile dysfunction, loss of sexual desire), headaches etc.

Most common respiratory disorders that are now linked with OSAS are COPD and bronchial asthma, and this association is also known as 'Overlap' and 'Alternate overlap syndrome', respectively. There is a positive association between OSAS and occasional wheezing, persistent wheezing, snoring, and a history of asthma. There are several mechanisms for how asthma may worsen OSAS and, conversely, how OSAS may complicate asthma.' Asthmatics have disrupted sleep architecture as a result of frequent arousals from sleep, decreases in sleep efficiency, and variable effects on sleep stage distribution when compared to healthy controls. There are certain factors that are responsible for increased prevalence of OSAS among asthma patients and these includes allergic rhinitis, oral and inhaled corticosteroid, gastro-eosophageal reflex disease (GERD), beta-2 receptor down regulation, decreased inspiratory muscle activity and neurohormonal changes ${ }^{9}$ etc. Prevalence of OSAS among asthma patients ranges from $27 \%$ to $60 \%$ world- wide. ${ }^{10,11}$ A high OSA risk of $39 \%$ was found in asthma patients by Auckley et al $^{12}$ and Alkhalil et al ${ }^{13}$ also found high prevalence of OSA in asthma group compared to control group ( $60 \% \mathrm{v} / \mathrm{s} 17 \%)$. A cohort study observed high risk of OSA in asthma patients compared to general population ( $12.1 \mathrm{v} / \mathrm{s} 4.84$ per 1000 person-year). ${ }^{14}$

Overlap patients (OSAS with COPD) presents with more nocturnal desaturation and pulmonary hypertension than patients with either OSAS or COPD alone. These patients had a higher breathing frequency, lower tidal volume, lower FEV1 and FVC, poor ventilatory response and reduction in respiratory drive than OSAS alone patients. ${ }^{15}$ COPD has been linked with skeletal-muscle myopathy and it may be that COPD (or cigarette smoking) affects the upper-airway dilator muscles or reflexes. The most significant sleep abnormality associated with COPD is nocturnal oxygen desaturation. Even without any upperairway abnormality, $27 \%$ to $70 \%$ of patients with COPD with oxygen saturation of $90 \%$ to $95 \%$ at room air experience substantial desaturation during night and particularly in REM phase of sleep. ${ }^{16}$ In addition to increased morbidity and mortality, patients with the overlap syndrome also have significantly worse quality of life when compared to 'COPD only' controls.
The prevalence of OSAS is known to be increasing in patient having idiopathic pulmonary fibrosis (IPF), therefore the most recent IPF guidelines recognize OSAS as an important associated comorbidity that can influence patient survival in these patients. ${ }^{17}$ Sleep is found to be markedly disturbed in several studies among IPF patients. Common abnormalities recognized are atypical sleep macro and micro architecture (increase stage 1 sleep, decrease REM, decrease sleep efficiency, increase arousal index etc), respiratory breathing pattern, oxygen desaturation and features of hypoventilation. The role of underlying OSAS in general health status of IPF patients has now been recognized in several studies. Some features of OSAS such as increased gastro esophageal reflux, exaggerated intra thoracic mechanical strain, and intermittent hypoxia with oxidative stress may impact progression of IPF. The associated cardiovascular and cerebrovascular morbidity seen in OSAS patient may turn IPF patients towards life threatening situation. The current recommendation favors immediate referral of IPF patient to exclude associated comorbidities such as OSAS and in high risk patient not only for better management strategy in such patient but inclusion in clinical trials and research as well. ${ }^{18}$

\section{OSAS and Cardiovascular disorders:}

Cardiovascular comorbidities are commonly and most frequently associated with OSAS. OSAS is an independent risk factor for the development of several cardiovascular consequences. The mechanisms involved in this association are rather complex and diverse. Patients with OSAS experience repetitive episodes of deoxygenation and reoxygenation during transient cessation of breathing that may provoke systemic effects. Alternate hypoxia and reoxygenation leads to endothelial dysfunction, oxidative stress and increased sympathetic activity during sleep. Levels of nitric oxide, a major vasodilator substance released by the endothelium, have been found to be decreased in OSAS patients. ${ }^{19}$ Several studies have reported higher level of a potent vasoconstrictor i.e. endothelin-1 in OSAS patients. ${ }^{20}$ Clinic-based studies have also suggested that OSAS is associated with impaired brachial artery flow-mediated dilation and endothelial-mediated vasodilatation impairment. $^{21}$

The largest epidemiological community-based study of OSAS and cardiovascular disease was the Sleep Heart 
Health Study, conducted on 6424 subjects across the USA. This study found that apnea-hypopnea index (AHI) $>11$ /hour was associated with 1.42 fold higher risk of development of any cardio vascular co morbidity as compare to AHI $<1.4 /$ hour. $^{22}$ OSAS may contribute to left ventricular failure, systemic hypertension, pulmonary hypertension, stroke and cardiac arrhythmias. Sympathetic discharge, mechanical stress on the myocardium from the intra thoracic pressure changes are potentially arrhythmogenic. Brady-arrhythmias are commonly encountered in OSAS and may correlate with the severity of disordered breathing. A prospective study demonstrated that the recurrence of atrial fibrillation at 12 months following successful cardioversion was halved for those treated for OSAS compared with untreated OSAS. $^{23}$

Observational studies have shown that hypertension and OSA often coexist and subjects with OSA tend to have higher blood pressure (BP) than matched controls. Longitudinal studies from the Wisconsin Sleep Cohort, which provides prospective evidence also implicates OSA as a possible causal factor in hypertension. ${ }^{24}$ Several studies have investigated the association between stroke and sleep-disordered breathing. Reports from two observational cohorts showed moderate to severe sleepdisordered breathing as risk factor for prevalent stroke and, with serial polysomnographic data, demonstrated that the preexisting sleep disorder may be a risk factor for incident stroke. ${ }^{25}$ Recently, there is increasing interest in the role of OSA treatment on outcomes in heart failure also.

A link between OSAS and peripheral artery disease (PAD) is also recognized. OSAS is known to be associated with several biologic pathways or development of atherosclerosis such as inflammation, systemic hypertension, endothelial dysfunction and insulin resistance. A multiethnic study of atherosclerosis (MESA) assessed association between OSAS and peripheral artery disease on 4,000 patients. Higher prevalence of PAD was observed in African American patient's compare to other ethnic groups. In longitudinal analysis; self reported OSAS was associated with two fold increase in newly diagnosed PAD measured by ankle brachial index. ${ }^{26}$ Therefore, one should recognize the risk of this association while dealing such patients.

\section{OSAS and Cognition:}

OSAS is associated with impairment of cognition, emotional state, and quality of life. The effects are most apparent in the severe cases. Reported impairments include global intellectual dysfunction and deficits in vigilance, alertness, concentration, short and long-term memory, and executive and motor function. Clinical studies suggest that OSAS impairs the structural integrity of several brain regions, including the medial temporal lobe. OSAS and hypertension trigger hypo perfusion and hypo metabolism at certain cerebral regions. OSAS promotes hippocampal atrophy, which is associated with memory impairment. ${ }^{27}$ A study done over 10 years found that patients with spontaneous CSF leak were associated with high rate of OSAS and hypertension. ${ }^{28}$ There are certain mechanisms that explain the impaired cognition among OSAS patients. Excessive daytime sleepiness increases the risk for impairment in multiple domains of cognition. ${ }^{29}$ Another possible hypothesis includes intermittent hypoxemia, sleep fragmentation and disturbed circadian rhythm. ${ }^{30}$

\section{OSAS and Epilepsy:}

Sleep disordered breathing (SDB) seems to be correlated with seizure disorders. Seven out of 11 subjects with incomplete seizure control were found to have mild to severe OSAS in a study. ${ }^{31}$ Multiple mechanisms may account for the higher than expected co-occurrence of epilepsy and SDB. OSAS may hinder the control of epilepsy through sleep disruption or repetitive hypoxemia. Generalized or focal seizures can induce central as well as obstructive apneas during the ictus. ${ }^{32} \mathrm{~A}$ common hypothesis underlying seizures, reduced central respiratory drive and even depression, may be represented by a dysfunction of serotonin pathways in the central nervous system. This could also have important implications for the sudden unexpected death in epilepsy. ${ }^{33}$ Some observational studies have suggested improvement of seizure control following SDB treatment by CPAP.

\section{OSAS and Neuromuscular disorders:}

Sleep-disordered breathing in neuromuscular disorders is due to an exaggerated reduction in lung volumes during supine sleep and specific features of the diseases that may promote upper airway collapse. Diaphragm palsy, spinal cord trauma, post polio syndrome, myasthenia gravis etc leads to obstructive as well as central sleep apnea. Decrease in the rib cage during REM sleep resulting in saw-tooth oxygen desaturation possibly represents the 
earliest manifestation of respiratory muscle weakness. Hypoventilation can occur in REM sleep and progress into non-REM sleep, with continuous desaturation and hypercarbia. Specific characteristics of neuromuscular disorders, such as pharyngeal neuropathy or weakness, macroglossia, bulbar manifestations, or low lung volumes, predispose patients to the development of obstructive events. $^{34}$

\section{OSAS and Metabolic syndrome/ endocrine disorders:}

Metabolic syndrome is an emerging public health problem characterized by hypertension, insulin resistance, dyslipidemia and obesity. Metabolic syndrome with cluster of cardiovascular risk factors is also associated with obstructive sleep apnea. Its prevalence varies from $74 \%$ to $85 \%$ among patients with OSAS. The metabolic syndrome is featured by a prothrombotic state with increased plasminogen activator inhibitor-1, fibrinogen and C-reactive protein (CRP). A number of epidemiological studies have found links between OSAS and metabolic syndrome. Obese OSAS patients may have an increased rate of metabolic syndrome and higher levels of serum lipids, fasting glucose, leptin, and fibrinogen than obese subjects without OSAS. ${ }^{35}$ Both OSAS and metabolic syndrome may exert negative synergistic effects on the cardiovascular system through multiple mechanisms. Many studies have shown that treatment of OSAS leads to improvement in hypertension and cholesterol level. ${ }^{36}$ Therefore, clinicians need to be encouraged to systematically evaluate the presence of metabolic abnormalities in OSAS and vice versa.

Some autoimmune diseases such as Hashimoto's thyroiditis are also linked with OSAS even if they are euthyroid. The mechanism proposed to explain this relationship are mucoprotien deposition in upper airway, decreased neural output to upper airway musculature, obesity and abnormal ventilator control etc. ${ }^{37}$

\section{OSAS and Depression:}

There is increasing prevalence of depression in OSAS patients that varies from $17 \%$ to $58 \%$. Many patients with OSAS are diagnosed to be having a depressive disorder and many receive antidepressant medications before they are actually referred for assessment for sleep disorder. Several studies attempted to delineate the relationship between depression and components of OSAS. One study found that $56.7 \%$ of OSAS patients had depressive symptoms and among them $21.6 \%$ had moderate to severe symptoms. ${ }^{38}$ Most common cause of depression in OSAS is sleep fragmentation and hypoxia. In some cases, treatment of comorbid insomnia and anxiety with a benzodiazepine and hypnotics may actually worsen OSAS. These medications may decrease muscle tone in the already functionally impaired upper airway dilator muscles, blunt the arousal response to hypoxia and hypercapnea, and increases the arousal threshold for the apneic event, therefore increasing the number and duration of apneas. ${ }^{39}$ Another study found that patients with treatment resistant depression with comorbid OSAS got improvement in mood and cognition after two month of CPAP therapy. ${ }^{40}$

\section{OSAS and Sexual life:}

Snoring is estimated to be the third most common cause of divorce in the United States and Great Britain. OSAS can cause erectile dysfunction (ED) in men and loss of libido in women. There are several mechanisms by which sleep apnea could cause ED, such as sleep fragmentation that reduces spontaneous erections at night, hormonal impairment (testosterone hormone level increase with sleep) or endothelial dysfunction. Depression is one of the important causes for decrease libido. ${ }^{41}$ On the other hand, oxidative stress affect hypothalamic-pituitary complex and lead to psychological depression and decreased libido. A study on 90 patients evaluated relationship between OSAS by PSG and ED by questionnaire and it was found that patients with OSAS were linked with low ED score and showed significantly improvement after CPAP therapy. ${ }^{42}$

OSAS has now been recognized as an important underlying pathogenic factor for sexual dysfunction. There is strong evidence to suggest that OSAS independently causes endothelial dysfunction which is link with ED. ED patients treated with CPAP has shown positive improvement after three months in few studies. ${ }^{43}$ OSAS is also linked with female sexual dysfunction irrespective of severity of OSAS. OSAS in female corresponds with pre and post menopausal women. Post menopausal women diagnose to have polycystic ovary syndrome (PCOS) that is characterized by high androgen level and low estrogen level is also linked with OSAS. PCOS is usually accompanied by certain characteristics such as insulin resistance, glucose intolerance, and type-2 diabetes, which can be linked to the onset of OSA. ${ }^{43}$ 


\section{OSAS and Renal disorders:}

A high percentage of OSAS patients suffer from urinary symptoms such as frequency, nocturia, enuresis and overactive bladder etc. There is also high risk for chronic kidney disease in patient having severe OSAS without hypertension and diabetes. End stage renal disease (ESRD) is believed to increase OSAS severity by mechanism such as fluid overload causing congestive cardiac failure. ESRD may also contribute to pathogenesis of OSAS. ${ }^{44}$ Intermittent hypoxia in OSAS patient results in oxidative stress that can cause alteration in bladder, detrusor instability and spontaneous contraction. Patient of benign prostate enlargement who frequently awake during sleep may have possible OSAS component. Severe OSAS has also been link with overactive bladder, urgency incontinence and nocturnal urination and altered bladder function in several studies. ${ }^{45}$

Both obstructive and central sleep apneas are not uncommon and equally distributed in patients of renal failure. Upto $80 \%$ of chronic dialysis patients complain of sleep disturbances and reduced daytime alertness. Most common cause of this condition is insulin resistance and atherosclerosis. Intermittent hypoxia and sympathetic activity also contribute it. In healthy young subjects, restricting time in bed to four hour per night for six days induces alterations in metabolic and endocrine function, including increased sympathetic tone and a state of insulin resistance. $^{46}$ Both abnormalities are well known complications of chronic uremia. There are few valid studies documenting the occurrence of sleep apnea among dialysis patients. As sleep apnea is common in renal failure, the nephrologists must have a high degree of suspicion in patients complaining of the symptoms or presenting with signs and symptoms suggestive of sleep apnea. A study conducted on 254 patients with renal disease, sleep apnea was found in 57\% hemodialysis patients of ESRD. ${ }^{47}$

\section{OSAS and Liver disorders:}

OSAS is also possibly associated with non alcoholic fatty liver disease and liver fibrosis. Several studies have suggested the impact of OSAS on liver function. A study in patients with fatty liver disease and altered liver function found $\mathrm{AHI}>5$ in $50 \%$ and further $20 \%$ among them were having severe OSAS. ${ }^{48}$ Chronic intermittent hypoxia (mimicking the oxygen profile of patients with severe OSAS) in mice on a high fat diet has been demonstrated to induce progression of hepatic steatosis to liver fibrosis and worsened hepatocellular injury. Hypoxia inducible factor-1 and over expression of lipo oxygenase has been linked with liver fibrosis in OSAS patients. ${ }^{49}$

\section{OSAS and Ocular manifestations:}

A growing body of literature shows a relationship between OSA and various ocular problems, however; the possible link between OSA and ocular manifestations are mostly depending on the observational and case series. Ocular associations of OSAS have been an issue of great interest in view of the irreversible complications it may cause and may have been preventable, if the OSAS is diagnosed and managed effectively beforehand or even after the diagnosis of the ocular findings. Ocular manifestations associated with OSAS are floppy eyelid syndrome, glaucoma, papilledema, retinal vein occlusion and nonarteritic anterior ischemic optic neuropathy, cornea disorders (keratoconus), central serous chorioretinopathy etc. Those who had been diagnosed with OSAS were 1.67 times more likely to have open angle glaucoma in the five years after their diagnosis than those without sleep disorder in one study. Treatment of OSAS has been shown to be effective in controlling the risk of glaucoma. ${ }^{50}$ There is a need for prospective randomized clinical trials and experimental studies to explain the underlying mechanisms of the associations between OSA and the related ocular pathologies.

\section{OSAS and Rheumatic disorders:}

Sleep abnormalities have been recognized in several rheumatic diseases including rheumatoid arthritis, osteoarthritis, systemic lupus erythematosis, systemic sclerosis, scleroderma, sarcodosis, behcet's syndrome etc. A large population study found $50 \%$ of rheumatoid arthritis patients having high risk for sleep apnea on standard questionnaire. OSAS in rheumatic patient may contribute to increase pain and fatigue perception. Untreated OSAS with intermittent hypoxia is associated with increase level of systemic inflammatory markers such as CRP and pro inflammatory cytokines. ${ }^{51}$ However, the impact of OSAS as comorbidity in rheumatic patients to the extent and response to therapy of inflammatory rheumatic disease is currently unknown.

\section{OSAS and Dental disorders:}

Many studies have shown greater prevalence of periodontitis in patients with diabetes, cardiovascular 
disease, rheumatoid arthritis, and osteoporosis. Research indicates that patients with sleep apnea have increased plasma markers of systemic inflammation and increased levels of circulating cytokines in obesity. It is hypothesized that OSAS may be linked to increased risk of periodontal disease because of its association with an elevated inflammatory response. ${ }^{52} \mathrm{~A}$ cross-sectional study on 687 subjects concluded that $17.5 \%$ of subjects had periodontitis, $46.6 \%$ had OSAS and $60 \%$ of those diagnosed with periodontitis also had OSAS. ${ }^{53}$

\section{OSAS and Skin disorders:}

Few studies have investigated a possible relationship between OSAS and dermatologic illnesses i.e. psoariasis, a chronic immune mediated inflammatory skin disease that is thought to be associated with OSAS, metabolic syndrome and cardiovascular disease. The link between OSAS and psoriasis is complex and possibly mediated by systemic inflammation. ${ }^{54}$ Few reports have also investigated the association between OSAS and atopic dermatitis. A retrospective cohort study from Taiwan with over 5.5 year follow up, investigated the relationship between OSAS and risk of atopic dermatitis. The incident rate for atopic dermatitis in patient with OSAS was 9.81 per 1000 person-years. The incidence was 1.5 times higher in patient with OSAS then age and gender matched comparison group. The risk was more pronounced in younger age group and in men. ${ }^{55}$ Further, studies are required to explore the underlying mechanism in this relationship.

\section{OSAS and ICU care:}

Sleep related breathing disorders are not uncommon in critical ill as well as in post operative patients because of anatomical reason there is increase risk of difficulty in endotracheal intubation. OSAS patients undergoing surgery are at more risk of post operative complications. Therefore high post operative transfer to ICU and increased stay in ICU is expected in OSAS patients. Minimal use of sedative and opioid as well as liberal use of CPAP is recommended in patients who have OSAS or having high risk of OSAS. There are several thumb rules to be remembered by critical care physician such as desaturation and apnea may not always be a part of respiratory failure, early positive airway pressure can be applied to prevent reintubation and extubation to be performed in lateral or semi upright position without use of sedatives. Regional anesthesia for surgery is better alternative in patient having OSAS. ${ }^{56}$

\section{OSAS in Children:}

Obstructive sleep disordered breathing is not uncommon in children. From $3 \%$ to $12 \%$ of children snore, while obstructive sleep apnea syndrome affects $1 \%$ to $10 \%$ of children. The majority of these children have mild symptoms, and many outgrow the condition. Consequences of untreated obstructive sleep apnea include failure to thrive, enuresis, attention deficit disorder, behavior problems, poor academic performance, endocrine, metabolic and cardiopulmonary disease. The most common etiology of obstructive sleep apnea in children is adenotonsillar hypertrophy and loss of neuro muscular tone. Snoring and mouth breathing often prompt parents to seek medical attention for their children. Septal deviation, choanal atresia, nasolacrimal cysts, and nasal aperture stenosis must be considered in infants. In older children, nasal polyps and turbinate hypertrophy must be ruled out. In children (1tol2 years), an apnea-hypopnea index greater than 1 (average: 0.1 to 0.5 events per hour) or a minimum oxygen saturation of less than $92 \%$ (average: $96 \% \pm 2 \%$ ) is considered abnormal. Adenotonsillectomy, a routine procedure, has been shown to improve snoring, OSAS, weight problems, enuresis, and behavior problems in children who have the entire clinical spectrum of sleep disordered breathing. CPAP is the treatment of choice when adenotonsillectomy is contraindicated or has failed. Children with craniofacial syndromes, neuromuscular diseases, medical comorbidities, or severe obstructive sleep apnea, and those younger than three years are at increased risk of developing postoperative complications and should be monitored overnight in the hospital. ${ }^{57}$

\section{OSAS and Cancer:}

Obstructive sleep apnea (OSA) has been associated with increased cancer mortality and cancer incidence. OSA has been recognized as an oxidative stress disorder. Chronic and intermittent hypoxia along with reactive oxidative stimuli can activate transcription factors, such as hypoxia inducible factor- 1 which are known to play a key role in regulating the various stages of tumor formation and progression. Sleep apnea related hypoxia may also increase the susceptibility to develop a new cancer. Increased overnight hypoxia as a surrogate of OSA severity was associated with increased cancer incidence in a multicenter, clinical cohort study between year 2003 and year 2007 at seven Spanish teaching 
hospitals. The findings of this study showed that OSA severity was independently associated with increased risk of incident cancer especially among male and in patients younger than 65 years of age. ${ }^{58}$

\section{OSAS and Road traffic accidents:}

It is widely assumed that the risk of automobile accidents is increased in patients with sleep apnea syndrome particularly in those with severe disease. Howard et $\mathrm{al}^{59}$ estimated that $50 \%$ among 3000 commercial drivers were at risk for sleep apnea. Assessment of the risk of OSAS and development of effective methods to identify and treat commercial drivers with OSAS is an important part of the mission of the certain motor carrier safety department in western countries. A study ${ }^{60}$ with 60 control and 60 cases of OSAS in motor drivers found that prevalence of at least one accident in last three years were higher in case group (33\%) than control group (18\%). Patients with sleep apnea, as confirmed by PSG had a greater probability of having a traffic accident than patients without sleep apnea. Drivers with an AHI above 15 were significantly more likely to have multiple accidents in a period of five years than drivers with AHI of less than five. ${ }^{61}$

\section{OSAS and Occupational hazards:}

Although, OSAS is a non-occupational disease, its frequency, comorbidities, and potential to impair performance, makes it an important determinant to the health and safety at the workplace. OSAS mostly remains undiagnosed in general population and at the work place as well and thus, remains untreated. Excessive daytime sleepiness related to OSAS has been repeatedly implicated in major transport accidents. OSAS has also been demonstrated to have adverse impacts on employees, healthcare costs and workplace productivity. ${ }^{62}$ Results from 27 observational studies ( $n=268,332$ ), suggest that workers with sleep problems had a 1.62 times higher risk of being injured than workers without sleep problems. Approximately $13 \%$ of work injuries could be attributed to sleep problems. ${ }^{63}$

\section{OSAS and Cellular phones:}

Cellular phone has become an integral part of our daily life. It's very difficult for today's youth to live a single day without using this technology as there is irresistible desire for its use. Use of mobile phones during late night can have profound effects on sleep, resulting in fragmented sleep and decreased grade point average, alertness, impaired concentration, depression, anxiety etc. It has been reported that about $25 \%$ to $47 \%$ of college students experience disruptions in sleep due to cellular phones. ${ }^{64}$ OSAS is becoming more prevalent in younger populations and is commonly under diagnosed in college aged students. In a sample of over 1,845 college students, over 500 students were at risk for at least one sleep disorder, with OSAS being the commonest one. ${ }^{65}$ In a sample of 60 college students, findings revealed that students who attended to technology after sleep onset reported increased sleep disruption and daytime impairment as compared to non-technology users. ${ }^{66}$

\section{CONCLUSION}

Within depth review of the systemic manifestations or associations of OSAS; authors of this paper stress on high degree of suspicion and the attention among all medical persons whether general physician, cardiologist, pulmonologist, surgeon, dentist, psychiatrist, neurologist, dietician, endocrinologist, pediatrician, otorhinolaryngologist, critical care and intensivist, nephrologists, hepatologist, ophthalmologists etc towards the OSAS as this is an important, yet under diagnosed but treatable disorder. Initially this disorder was linked to only few sleep disturbances but huge literatures now available prove that OSAS is widely connected in human body. It can affect major organs to minute capillaries, old person to neonate and even young and active teenage, a simple home resident people to active workplace person, non obese person to obese person and therefore represents a truly complex syndrome. Therefore, treating physician should always try to assess the sleeping pattern and behavior in patients with even slightest doubt of OSAS. Several studies have proved that treatment of OSAS by CPAP significantly improves not only the sleeping problem but also the associated comorbid illness.

\section{REFERENCES}

1. Lee W, Nagubadi S, Kryger MH, Mokhlesi B. Epidemiology of obstructive sleep apnea: A populationbased perspective. Expert Rev Respir Med 2008; 2: 34964.

2. Punjabi NM. The epidemiology of adult obstructive sleep apnea. Proc Am Thorac Soc 2008; 5:136-43.

3. Franklin KA, Lindberg E. Obstructive sleep apnea is a common disorder in the population - Areview on the epidemiology of sleep apnea. J Thorac Dis 2015; 7: 1311-22.

4. Prasad CN. Obstructive sleep apnea hypopnea syndromeIndian scenario. Perspective in medical research 2013; 1: 22-25. 
5. Carskadon MA, Dement WC. Normal human sleep: An overview. Principles and Practice of Sleep Medicine, 4th ed. Philadelphia, Elsevier Saunders, 2005.

6. Casale M, Pappacena M, Rinaldi V, Bressi F. Obstructive sleep apnea Syndrome: From phenotype to genetic basis. Current Genomics 2009; 10: 119-26.

7. Lam JCM, Sharma SK, Lam B. Obstructive sleep apnoea: definitions, epidemiology and natural history. Indian $\mathrm{J}$ Med Res 2010; 131: 165-70.

8. Lawati NM, Patel SR, Ayas NT. Epidemiology, risk factors and consequences of obstructive sleep apnea and short sleep duration. Prog Cardiovasc Dis 2009; 51: 285 93.

9. Michael E, Jonathan P, John G. Sleep and obstructive lung diseases. Sleep Med Clin 2008; 3(4): 505-15.

10. Teodorescu M, Polomis DA, Hall SV, Gangnon BE, Peterson AG. Association of obstructive sleep apnea risk with asthma control in adults. Chest 2010; 138(3): 543-50.

11. Zidan M, Daabis R, Gharraf H. Overlap of obstructive sleep apnea and bronchial asthma: Effect on asthma control. Egyptian J Chest Dis Tuberc 2015; 64: 425-30.

12. Auckley D, Moallem M, Shaman Z, Mustafa M. Findings of a Berlin questionnaire survey: Comparison between patients seen in an asthma clinic versus internal medicine clinic. Sleep Med 2008; 9: 494-99.

13. Alkhalil M, Schulman E, Getsy J, Obstructive sleep apnea syndrome and asthma: what are the links? J Clin Sleep Med 2009; 5: 71-78.

14. Shen TC, Lin CL, Wei CC, Chen $\mathrm{CH}$, et al. Risk of obstructive sleep apnea in adult patients with asthma: a population-based cohort study in Taiwan. PloS One 2015; 10(6): e 0128461.

15. Zamarrón C, Paz VG, Morete E, Matías FC. Association of chronic obstructive pulmonary disease and obstructive sleep apnea consequences. Int J COPD 2008; 3(4): 671-82.

16. Lewis CA, Fergusson W, Eaton T, Zeng I, et al. Isolated nocturnal desaturation in COPD: prevalence and impact on quality of life and sleep. Thorax 2009; 64 (2):133-38.

17. Raghu G, Collard HR, Egan JJ, et al. An official ATS/ERS/JRS/ALAT statement: idiopathic pulmonary fibrosis: evidence-based guidelines for diagnosis and management.AmJRespir Crit Care Med 2011;183: 788-824.

18. Schiza S, Mermigkis C, Margaritopoulos GA, et al. Idiopathic pulmonary fibrosis and sleep disorders: no longer strangers in the night. Eur Respir Rev 2015; 24: 327-39.

19. Oyama J, Yamamoto $\mathrm{H}$, Maeda $\mathrm{T}$, et al. Continuous positive airway pressure therapy improves vascular dysfunction and decreases oxidative stress in patients with the metabolic syndrome and obstructive sleep apnea syndrome. Clinical Cardiology 2012; 35 (4): 231-36.
20. Saarelainen S, Hasan J. Circulating endothelin-1 and obstructive sleep apnoea. Eur Respir J 2000; 16(4): $794-$ 95.

21. Itzhaki S, Lavie L, Pillar G, et al Endothelial dysfunction in obstructive sleep apnea measured by peripheral arterial tone response in the finger to reactive hyperemia. Sleep 2005; 28: 594-600.

22. Shahar E, Whitney CW, Redline S, et al. Sleep-disordered breathing and cardiovascular disease: cross-sectional results of the Sleep Heart Health Study. Am J Respir Crit Care Med 2001; 163: 19-25.

23. Kanagala R, Murali NS, Friedman PA, et al. Obstructive sleep apnea and the recurrence of atrial fibrillation Circulation 2003; 107:2589-94.

24. Peppard PE, Young T, Palta M, Skatrud J. Prospective study of the association between sleep-disordered breathing and hypertension. N Engl J Med 2000; 342: 1378-84.

25. Arzt M, Young T, Finn L, Skatrud JB, Bradley TD. Association of sleep disordered breathing and the occurrence of stroke. Am J Respir Crit Care Med 2005; 172: 1447-51.

26. Nagayoshi M, Lutsey PL, Benkeser D, Wassel CL, Folsom AR, Shahar E et al.Association of sleep apnea and sleep duration with peripheral artery disease: The MultiEthnic Study of Atherosclerosis (MESA). Atherosclerosis 2016; 251:467-75.

27. Daulatzai MA. Evidence of neurodegeneration in obstructive sleep apnea: Relationship between obstructive sleep apnea and cognitive dysfunction in the elderly. J Neurosci Res 2015; 93(12): 1778-94.

28. Nelson RF, Gantz BJ, Hansen MR. The rising incidence of spontaneous cerebrospinal fluid leaks in the United States and the association with obesity and obstructive sleep apnea. Otol Neurotol 2015; 36(3): 476-80.

29. Ohayon MM, Vecchierini MF. Daytime sleepiness and cognitive impairment in the elderly population. Arch Intern Med 2002; 162:201-08.

30. Verstraeten E, Cluydts R. Executive control of attention in sleep apnea patients: Theoretical concepts and methodological considerations. Sleep Med Rev 2004; 8(4):257-67.

31. Chihorek AM, Abou-Khalil B, Malow BA. Obstructive sleep apnea is associated with seizure occurrence in older adults with epilepsy. Neurology 2007; 69: 1823-27.

32. Bateman LM, Li CS, Seyal M. Ictal hypoxemia in localization-related epilepsy: analysis of incidence, severity and risk factors. Brain 2008; 131:3239-45.

33. Richerson GB, Buchanan GF. The serotonin axis: Shared mechanisms in seizures, depression, and SUDEP. Epilepsia 2011; 52(1):28-38. 
34. Aboussouan LS. Sleep-disordered Breathing in Neuromuscular Disease. Am J Respir Crit Care Med 2015 ;191(9): 979-89.

35. Basoglu OK, Sarac F, Sarac S, Uluer H, Yilmaz C. Metabolic syndrome, insulin resistance, fibrinogen, homocysteine, leptin, and C-reactive protein in obese patients with obstructive sleep apnea syndrome. Annals Thorac Med 2011; 6: 120-25.

36. Sharma SK, Agrawal S, Damodaran D et al. CPAP for the metabolic syndrome in patients with obstructive sleep apnea. NEngl J Med 2011; 365: 2277-86.

37. Erden S, Cagatay T, Buyukozturk S, Kıyan E, Cuhadaroglu C. Hashimoto thyroiditis and obstructive sleep apnea syndrome: Is there any relation between them? Eur J Med Res 2004; 9: 570-72.

38. Sherbini AM, Bediwy AS, Mitwalli A. Association between obstructive sleep apnea (OSA) and depression and the effect of continuous positive airway pressure (CPAP) treatment. Neuropsychiatric Disease and Treatment 2011; 7: 715-21.

39. Guilleminault C. Benzodiazepines, breathing, and sleep. Am J Med 1990; 88(3A):255-85.

40. Habukawa M, Uchimura N, KakumaT, et al. Effect of CPAP treatment on residual depressive symptoms in patients with major depression and coexisting sleep apnea: contribution of daytime sleepiness to residual depressive symptoms. Sleep Med 2010; 11(6): 552-57.

41. Andersen ML, Santos-Silva R, Bittencourt LR, et al. Prevalence of erectile dysfunction complaints associated with sleep disturbances in Sao Paulo, Brazil: A population-based survey. Sleep Med 2010; 11: 1019-24.

42. Husnu T, Ersoz A, Bulent E, et al. Obstructive sleep apnea syndrome and erectile dysfunction: does long term continuous positive airway pressure therapy improve erections? African Health Sciences 2015; 15(1): 171-79.

43. Tasali E, Van Cauter E, Hoffman L, Ehrmann DA. Impact of obstructive sleep apnea on insulin resistance and glucose tolerance in women with polycystic ovary syndrome. J Clin Endocrinol Metab 2008; 93: 3878-84.

44. Lyons OD, Chan CT, Yadollahi A, Bradley TD. Effect of ultrafiltration on sleep apnea and sleep structure in patients with end-stage renal disease. Am J Respir Crit Care Med 2015; 191: 1287-94.

45. Dagur G, Warren K, Ambroise S, et al. Urological manifestations of obstructive sleep apnea syndrome: A review of current literature. Transl Biomed 2015, 6:3.doi: 10.21767/2172-0479.100022.

46. Spiegel K, Leproult R, Van Cauter E: Impact of sleep debt on metabolic and endocrine function. Lancet 1999; 354: 1435-39.
47. Nicholl DD, Ahmed SB, Loewen AH, et al. Declining kidney function increases the prevalence of sleep apnea and nocturnal hypoxia. Chest 2012; 141: 1422-30.

48. Petta S, Marrone O, Torres D, et al. Obstructive sleep apnea is associated with liver damage and atherosclerosis in patients with non-alcoholic fatty liver disease. PLOS ONE 2015; DOI:10.1371/journal.pone.0142210: 1-15.

49. Mesarwi OA, Shin MK, Fonti SB et al. Hepatocyte hypoxia inducible factor-1 mediates the development of liver fibrosis in a mouse model of nonalcoholic fatty liver disease. PLOS ONE 2016; DOI: 10.1371/ journal.pone.0168572: 1-15.

50. Arda H, Sevim DG, Mirza E, et al. Ocular manifestations of obstructive sleep apnea. Austin J Sleep Disord 2015; 2(2): 1011.

51. Taylor-Gjevre RM, Nair BV, Gjevre JA. Obstructive sleep apnoea in relation to rheumatic disease. Rheumatology 2013; 52: 15-21.

52. Gunaratnam K, Taylor B, Curtis B, Cistulli P. Periodontitis and sleep apnoea. Ann R Australas Coll Dent Surg 2008; 19:48-49.

53. Seo WH, Cho ER, Thomas RJ, et al. The association between periodontitis and obstructive sleep apnea: a preliminary study. J Periodontal Res 2013; 48(4): 500-06.

54. Yang YW, Kang JH, Lin HC. Increased risk of psoriasis following obstructive sleep apnea: a longitudinal population-based study. Sleep Med 2012; 13:285-89.

55. Tien KJ, Chou CW, Lee SY, Yeh NC, Yang CY, et al. Obstructive sleep apnea and the risk of atopic dermatitis: a population-based case control study. PLoS ONE 2014; 9(2): e89656. doi:10.1371/journal.pone.0089656.

56. Steiner S, Keymel S. Sleep related breathing disorders in an intensive care setting. Clin Res Pulmonol 2015; 3(1): 1032.

57. Chan J, Edman JC, Koltai PJ, et al. Obstructive sleep apnea in children. Am Family Physician 2004; 69(5): 1147-54.

58. Campos-Rodriguez F, Martinez-Garcia MA, Martinez M, Duran-Cantolla J, Peña Mde L, Masdeu MJ, et al. Association between obstructive sleep apnea and cancer incidence in a large multicenter Spanish cohort. Am J Respir Crit Care Med 2013; 187: 99-105.

59. Howard ME, Desai AV, Grunstein RR, et al. Sleepiness, sleep-disordered breathing, and accident risk factors in commercial vehicle drivers. Am J Respir Crit Care Med 2004; 170:1014-21.

60. Barbe F, Pericas J, Munoz A et al. Automobile accidents in patients with sleep apnea syndrome: An epidemiological and mechanistic study. Am J Respir Crit Care Med 1998; 158: 18-22.

61. Young T, Blustein J, Finn L, Palta M. Sleep-disordered 
breathing and motor vehicle accidents in a populationbased sample of employed adults. Sleep 1997; 20: 60813.

62. McKay MP. Fatal consequences: obstructive sleep apnea in a train engineer. Ann Fam Med 2015; 13: 583-6.

63. Uehli K, Mehta AJ, Miedinger D, et al. Sleep problems and work injuries: A systematic review and meta analysis. Sleep Medicine Reviews 2014; 18: 61-73.

64. Adams SK, Kisler TS. Sleep quality as a mediator between technology- related sleep quality, depression, and anxiety. Cyberpsychol Behav Soc Netw 2013; 16: 2530.

65. Gaultney JF. The prevalence of sleep disorders in college students: impact on academic performance. J Am Coll Health 2010; 59:91-97.

66. Adams SK, Kisler TS and Feragne RM. Daytime impairment due to college student technology use during sleep: a first step toward exploring similarities to obstructive sleep apnea. Austin J Sleep Disord 2015; 2(1): 1006.

\section{Corresponding Author}

Dr Ramakant Dixit, A-60, Chandravardai Nagar, Ajmer. Rajasthan, India.

e-mail : dr.ramakantdixit@gmail.com

\section{Miscellanea}

\section{Campus News}

\section{WORKSHOP}

Rajasthan University of Health Sciences conducted three days workshop on "Research Methodology - Basics of Biostatistics Workshop" from $9^{\text {th }}$ to $11^{\text {th }}$ February 2018. Eminent speakers like Dr N C Jain, Scientist G, ICMR, New Delhi; Dr Shreenivas, Professor, Biostatistics, AIIMS, Delhi; Dr Mario Vaz, Professor, Physiology, St John's Medical College, Bangaluru; Dr Tulsi Adhikari, Scientist-E, NIMS, ICMR, New Delhi; Dr Atul Juneja, Scientist-D, NIMS, ICMR, New Delhi and Dr V M Katoch, ICMR-NASI Chair at Rajasthan University of Health Sciences, Jaipur conducted the workshop. The workshop comprised of sessions on biostatistics, problem identification and formulation of research question/hypothesis, review of literature in developing research proposals, concept of sampling, data management, hypothesis testing, sample size calculation, representation of data, correlation and regression analysis. Hands on training was given to the participants on sampling, descriptive analysis and regression analysis. Workshop was attended by 55 participants from all over the state. Rajasthan Medical Council accredited 6 credit hours.

\section{CME PROGRAMMS}

A guest lecture on $9^{\text {th }}$ February 2018 on "Basic Research Methodology" was delivered at Rajasthan University of Health Sciences, Jaipur by Dr Mario Vaz, Professor, St John's Medical College, Bengaluru. 